\title{
A GAL4-UAS system for the worm community
}

A bipartite gene expression system optimized for C. elegans promises to improve efficiency and reproducibility for worm researchers.

Any biologist new to writing their own software quickly realizes the power of reusing someone else's established code: by mixing and matching functions that already work, you can quickly build up new and useful programs that are stable and robust.

For biologists that also work with model organisms, the same principle underlies the use of bipartite gene expression systems for creating transgenic animals: rather than creating a new transgenic animal from scratch every time you want expression of a certain transgene in a specific cell-type, you can take already established driver lines and cross them with animals from a desired effector line. Similar to opensource software for programmers, over time, as the number and diversity of driver and effector lines increases in a scientific community, they become powerful and indispensible tools.

Both the mouse and fly communities have widely adopted bipartite systemsCre-Lox in the mouse, and GAL4-UAS in flies-and have reaped significant advantages for creating and sharing genetic lines. With their recent paper in Nature Methods, Paul Sternberg at the California Institute of Technology and colleagues have now introduced a GAL4-UAS system specifically designed for C. elegans, which they call the cGAL system (Nat. Methods 14, 145-148; 2017). While the Sternberg lab hopes the system will provide the same combinatorial power the Drosophila field has enjoyed for years, they feel the system will also help significantly improve the reproducibility of results across labs by enabling worm researchers to reuse a common and large set of well-defined driver and effector strains.

Despite being a popular and established model organism for geneticists, it's proven difficult to develop a robust bipartite system

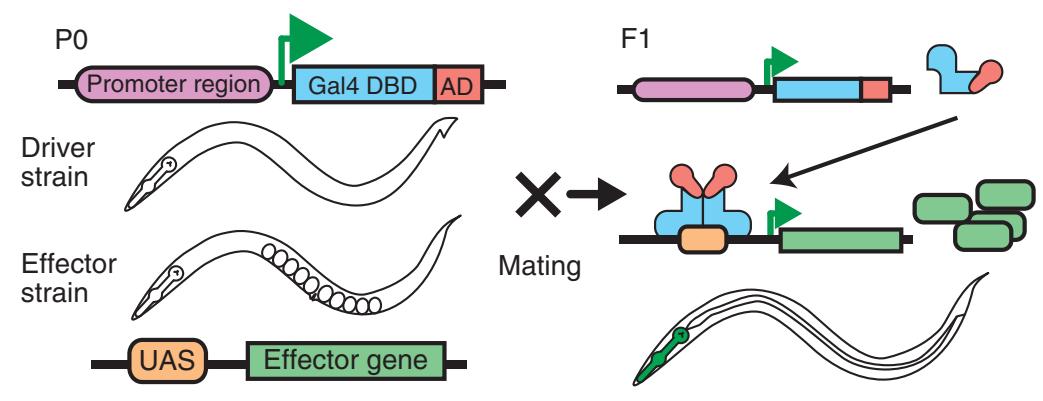

Schematic demonstrating the cGAL bipartite system, crossing a driver and effector line. Adapted from Nat. Methods 14, 145-148 (2017).

like GAL4-UAS for C. elegans. Gal4, which serves as the "driver" of cell-type specific gene expression, traditionally requires temperatures of $\sim 30^{\circ} \mathrm{C}$ to work well. Gal4, therefore, has worked great in Drosophila, which are typically grown at temperatures near $30^{\circ} \mathrm{C}$. But in worms, which are typically grown at $15-25^{\circ} \mathrm{C}$, the same Gal4 protein has failed to drive sufficient levels of transgene expression. According to Sternberg, "There have been probably a half dozen labs in the past that tried the GAL4-UAS system in worms, including ours, and it didn't work, or at least not in a way that was useable."

Knowing it would be a challenge, the two lead authors of the new paper, Han Wang and Jonathan Liu, dug in and went to work; their first step being to tackle the temperature problem. Instead of using Gal4 from Saccharomyces cerevisiae, which grows optimally at higher temperatures $\left(30-34^{\circ} \mathrm{C}\right)$, they used Gal4 from a more cryophilic yeast species (Saccharomyces kudriavzevii). Additionally, the team optimized the Gal4 activation domain, and also the UAS copy number (comparing 5X, 10X, 15X, and 20X copies) to increase expression levels. Testing GFP expression in several specific cell types, the paper demonstrates that the team's optimized cGAL system drives robust transgene expression in worms grown at their typical temperatures of $15-25^{\circ} \mathrm{C}$.
With the cGAL system in hand, as well as a starter kit of driver and effector strains, Sternberg's group now hopes to spread their adoption in the worm community to help improve efficiency and reproducibility. While some investigators may initially shy away from changing their old habits of "do it yourself" transgenics, by creating and sharing more strains the Sternberg lab hopes confidence and use in the strains will pick up. "I kind of think of it like software code, as it gets distributed and used more and more, all of the bugs and quirks come out", says Liu. "The next step is to provide a central location to make that kind of information easily accessible to the community, as the number of strains grows."

Although creating a transgenic worm from scratch is relatively easy-a potential argument against adopting the cGAL system-similar transgenics created from independent labs can have slight but important differences, which can create trouble when trying to understand and replicate subtle phenotypes. For Han, who has worked on C. elegans for years, first as a graduate student and now as a postdoc, it comes down to a simple question: "Do you want to stick to the old fashioned system you are used to, or do you want to try something that has the potential to be better?" Dustin M. Graham 